Introduction Aspirin acts as an anti-thrombotic drug by inhibiting platelet cyclooxygenase- 1 (COX-1) and blocking the production of thromboxane A2, but it also has off-platelet actions that may limit its effectiveness. Since mice have very marked differences in aspirin pharmacokinetics they cannot be used to explore the effects of low dose aspirin in humans. To address these questions, we developed platelet COX-1 knockout mice (platelet-COX-1 ${ }^{-/-}$) to recapitulate the effect of low-dose aspirin in humans.

Methods and Results. Cox $-1^{f l / f l}$ mice were paired with Pf4-Cre mice to delete COX-1 in the MK lineage. The absence of COX-1 in platelets was confirmed by Western blot analysis, confocal immunofluorescence microscopy and by analysing the distribution of the COX-1 signal and mass with an highthroughput confocal imaging system. The production of thromboxane $\mathrm{B} 2\left(\mathrm{TxB}_{2}\right)$ induced by collagen, $\mathrm{A} 23187$ and the PAR-4 activating peptide in platelet rich plasma and whole blood was significantly decreased in platelet-COX-1 ${ }^{-/}$mice. This effect was not further reduced by the addition of aspirin in vitro. Further eicosanomic analysis was performed using LC-MS/MS of samples from untreated and A23187-stimulated whole blood. 11- and 15-hydroxyeicosatetraenoic acid (HETE), alongside $\mathrm{TxB}_{2}$ formation were inhibited after platelet COX-1 deletion. Production of 12-HETE, coming from 12-lipoxygenase (LOX), and 5-HETE coming from leukocyte 5 -LOX, was unchanged in platelet-COX-1 $1^{-/-}$mice. To better dissect the effects of aspirin in vivo, we used a thrombosis model caused by ferric chloride injury in the mouse carotid artery. Platelet $\mathrm{COX}-1^{-/-}$mice, that recapitulate the effects of low-dose aspirin, formed unstable thrombi that were embolizing before producing a stable vessel occlusion. Interestingly, when a high dose of aspirin $(10 \mathrm{mg} / \mathrm{kg})$ was injected into the mice, the thrombus was stable and formed earlier than in the vehicle-treated mice.

Conclusions We have produced the first platelet-COX-1 ${ }^{-/}$ mouse and characterised its phenotype and eicosanomic profile. This model is allowing us to mechanistically dissect the platelet and non-platelet effects of aspirin and so better model the effects of clinically important anti-thrombotic therapy.

\section{PAEDIATRIC CARDIAC ARREST: IS THERE ANY MYOCARDIAL DAMAGE IN PAEDIATRIC PATIENTS WHEN ADULT DEFIBRILLATION ENERGIES ARE ADMINISTERED?}

Laura Davis*, ${ }^{1}$ Rebecca Funston, Paul Crawford, ${ }^{1}$ Olibhar McAlister, ${ }^{1}$ Ben McCartney, ${ }^{1}$ Hannah Torney, ${ }^{2}$ Alistair Courtney, ${ }^{3}$ Rachael Gregson, ${ }^{4}$ David McEneaney, ${ }^{3}$ Eddie Clutton, ${ }^{5}$ Jennifer Adgey. ${ }^{1}$ Physio-Control; ${ }^{2}$ Ulster University; ${ }^{3}$ University of Edinburgh; ${ }^{4}$ Craigavon Area Hospital; ${ }^{5}$ Royal Victoria Hospital

\subsection{6/heartjnl-2017-311726.147}

Purpose When attempting defibrillation in an out-of-hospital paediatric patient, the use of a reduced energy is currently recommended in the resuscitation guidelines due to concerns surrounding potential myocardial damage. The purpose of this pilot study was to investigate the 1 st shock success of two different automated external defibrillator (AED) energy protocols and observe the resulting levels of myocardial damage in a paediatric model of cardiac arrest.

Method A total of six piglets $(10-25 \mathrm{~kg})$ were anaesthetised, instrumented and ventricular fibrillation (VF) was electrically induced. After approximately $15 \mathrm{~s}$ of untreated VF, a defibrillation shock was delivered using the selected device in line with Protocol A (adult energy doses of 150J) or Protocol B (paediatric energy doses of 75J). Following successful defibrillation, a $3 \mathrm{~min}$ recovery period was allowed for the heart to recover before $\mathrm{VF}$ was again induced with a maximum of 20 shocks administered. Blood samples were collected at predefined time-points whilst the animal was under anaesthesia and analysed for cardiac troponin I (cTnI). After the defibrillation phase of the protocol, the animals remained anaesthetised and vital signs monitored for a period of 6 hours with blood samples collected hourly for further analysis.

Results A total of 120 shocks were delivered across both protocols with a 100\% shockable rhythm detection and 100\% first shock success observed in both Protocol A and B. Prior to the induction of VF, Protocol A and B presented with similar initial mean $( \pm S D)$ levels of cTnI; $0.04( \pm 0.03) \mathrm{ng} / \mathrm{ml}$ $(\mathrm{n}=3)$ and $0.03( \pm 0.02) \mathrm{ng} / \mathrm{ml}(\mathrm{n}=3)$, respectively. Comparable results of cTnI were also observed upon completion of the defibrillation phase (Shock 20); Protocol A - $0.12( \pm 0.05)$ $\mathrm{ng} / \mathrm{ml}$ and Protocol B $-0.14( \pm 0.07) \mathrm{ng} / \mathrm{ml}$. An increase in mean cTnI was detected into the rest period, peaking by 5 hours for both energy protocols. At the 6 hour endpoint of this study, the mean value of cTnI in the blood was reducing for both energy protocols with no statistically significant difference observed between the high and low defibrillation energy groups.

Conclusion There was no statistical evidence to suggest that treating the paediatric animals with a higher adult shock energy caused more myocardial damage than a paediatric shock energy. Further investigations are warranted to determine the long term impact on myocardial tissues in a paediatric patient.

\section{RAISED INTRACELLULAR CA ${ }^{2+}$ CONCENTRATION BY PERSISTENT NA ${ }^{+}$CHANNELS ACTIVATION DISRUPTS CELLULAR CIRCADIAN RHYTHMICITY OF BMAL1 CLOCK GENE IN CULTURED ATRIAL MYOCYTES}

Andria Siakalli* ${ }^{*}$ Daan van der Veen, Rita Jabr. University of Surrey

10.1136/heartjnl-2017-311726.148

One of the main contributor to atrial arrhythmias (AA) is the activation of the persistent $\mathrm{Na}$ channels $\left(\mathrm{Na}_{\mathrm{P}}\right)$ leading to raised intracellular $\mathrm{Ca}^{2+}$ concentration $\left(\left[\mathrm{Ca}^{2+}\right]_{\mathrm{i}}\right)$. Incidences of AA exhibit diurnal patterns suggestive of possible correlation between circadian rhythms and atrial electrophysiology. Circadian rhythms are mediated by cellular circadian clocks, made up of transcriptional-translational feedback loops consisting of several genes, in particular Bmal1. So far, the correlation between raised $\left[\mathrm{Ca}^{2+}\right]_{i}$ induced by activated $\mathrm{Na}_{\mathrm{P}}$ and Bmal1 rhythmicity is unknown. The aim of this study is to assess the effects of 1) $\mathrm{Na}_{\mathrm{P}}$ opener, ATXII, Anemonia Sulcata toxin II and 2)raised $\left[\mathrm{Ca}^{2+}\right]_{i}$ induced by $\mathrm{Na}_{\mathrm{P}}$ on Bmal1 gene circadian rhythmicity in cultured mouse atrial (HL-1-6) cells and mouse embryonic fibroblasts (MEFs, positive control).

HL-1-6 were grown in Claycomb medium with 10\% FBS, $0.1 \mathrm{mM}$ norepinephrine and $2 \mathrm{mM}$ l-glutamine, whereas, MEFs were maintained in DMEM with 10\% FBS. The cells were transduced with Bmal1::luciferase (BMAL1::LUC) probe to determine bioluminescence rhythmicity through long-term bioluminescence recording over several circadian cycles (45 days). Cells were serum shocked (2 hours) using 50\% FBS to synchronise all cellular clocks. Cells were then incubated in 
control media alone or with $1 \mathrm{nM}$ ATXII in presence/absence of either $10 \hat{\mathrm{I}}^{1 / 4} \mathrm{M}$ ranolazine (Ran; $\mathrm{Na}_{\mathrm{P}}$ blocker) or $5 \mu \mathrm{M}$ BAPTA-AM. All data were expressed as mean \pm SEM. Differences among groups were tested by ANOVA followed by Bonferroni post-hoccomparison. The null hypothesis was rejected at $\mathrm{p}<0.05$.

Under control conditions, HL-1-6 cells and MEFs exhibited 24 hours Bmal1 circadian rhythms with a period of 26.09 \pm 0.77 hours $(n=8)$ and $25.22 \pm 0.26$ hours $(n=8)$, respectively. The Bmal1 rhythmicity was highly disrupted in HL-1-6 cells only when incubated with ATXII, with a 10 hours shortening $(17.06 \pm 1.07$ hours; $n=8 ; p<0.05)$ in their period. The effect of ATXII on HL1-6 cells was reversed in presence of Ran (25.32 $\pm 0.97 ; \mathrm{n}=8 ; \mathrm{p}<0.05$ vs ATXII). In fura2-AM loaded cells, $\left[\mathrm{Ca}^{2+}\right]_{\mathrm{i}}$ was significantly increased with ATXII $(2.30$ $\pm 0.05)$ when compared to control $(0.96 \pm 0.08 ; \mathrm{n}=3 ; \mathrm{p}<0.01)$ and Ran fully reversed ATXII effect $(0.94 \pm 0.02 ; n=3$; $\mathrm{p}<0.01)$. Then, the effects of raised $\left[\mathrm{Ca}^{2+}\right]_{\mathrm{i}}$ by ATXII on Bmal1 rhythmicity was assessed using BAPTA-AM. Indeed, addition of BAPTA-AM prevented ATXII-induced Bmal1 period shortening (ATXII 17.09 $\pm 1.21 ;$ ATX+BAPTA-AM $29.91 \pm 0.54 ; \mathrm{n}=8 ; \mathrm{p}<0.05)$.

This study showed that in HL-1-6 cells only but not MEFs:1)ATXII treatment leads to a significant shortening of Bmal1 driven bioluminescence by almost 9 hours; 2)ATXII effect is fully reversed by ranolazine; 3)ATXII effects are mediated by raised $\left[\mathrm{Ca}^{2+}\right]_{\mathrm{i}}$ and hence was blocked by BAPTAAM. In conclusion, this study suggests a possible correlation between ATXII-induced atrial arrhythmias and Bmal1 gene and hence proposing a role for $\mathrm{Na}_{\mathrm{p}}$ activation channels in the disruption of atrial circadian rhythm.

\section{A HIGH THROUGHPUT SIRNA SCREENING IDENTIFIES RAC1 AS A POTENTIAL MODULATOR OF THE ENDOCYTOSIS OF THE TYROSINE KINASE RECEPTOR TIE2}

Marta Giralt-Pujol* ${ }^{*}$ Elizabeth Smythe. University of Sheffield

\subsection{6/heartjnl-2017-311726.149}

The Tie2 receptor is a cell surface tyrosine kinase receptor expressed almost exclusively in endothelial cells, where it is mainly implicated in angiogenesis. Indeed, Tie2 has been related to various pathologies with vascular defects such as pulmonary hypertension, diabetes retinopathy and tumour growth. Tie 2 activation and function is regulated in a complex manner by multiple factors that are still being investigated. For instance, after activation by the agonistic ligand Angiopoietin-1 (Ang1), Tie2 is internalised in cells by endocytic mechanisms that are yet to be fully characterised. As it has been shown that endocytosis can play a regulatory role in signalling, our hypothesis is that the endocytosis of Tie2 may also be important in the regulation of its activity and cellular output. Therefore, we decided to characterise the endocytic mechanisms involved in the internalisation of Tie2 to determine whether endocytosis can be a regulator of Tie2 signalling.

To facilitate the study of Tie 2 we created a HeLa cell line with inducible expression of a Tie $2^{\text {FLAG }}$ receptor that reflects expression levels of endogenous Tie2 in Human Umbilical Vein Endothelial Cells (HUVECs). HUVECs were isolated from umbilical cords anonymously donated in the Sheffield Hallamshire Hospital. To study the endocytosis of Tie2 receptor we developed a cell immunofluorescence-based internalisation assay to quantify the amount of internalised agonistic ligand Ang1 in a High Throughput Screening format (HTS).

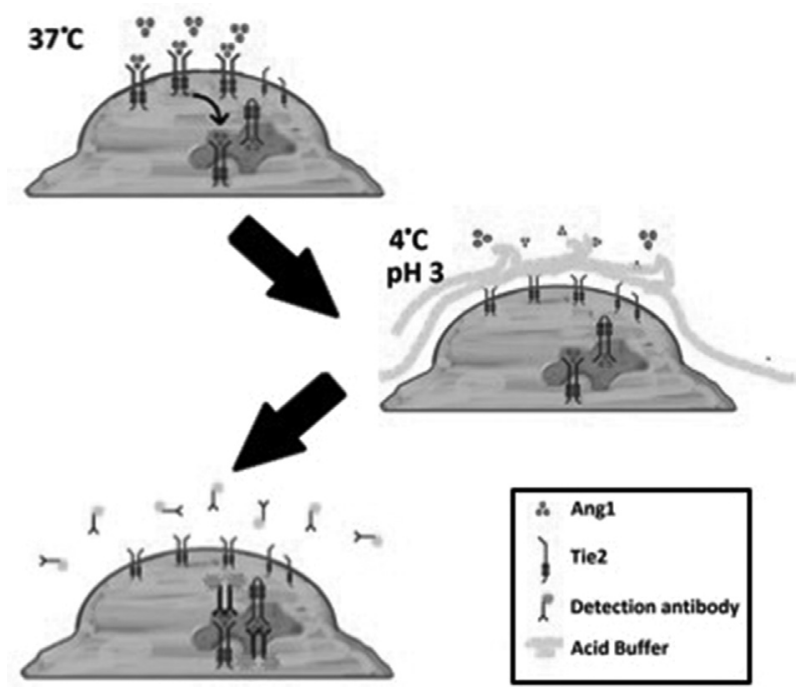

Abstract 150 Figure 1

Using the HTS internalisation assay we screened in HeLa/ Tie $2^{\text {FLAG }}$ cells a library of siRNAs targeting a selection of proteins involved in membrane trafficking. A selection of hits were selected for a secondary screen using different siRNAs and a pilot validation screen was performed using HUVECs. Interestingly, components of both clathrin-dependent and independent internalisation such as clathrin, AP2 subunits or caveolins were highlighted as potential regulators of the membrane trafficking of Tie2. Remarkably, the most robust hits along the different screens were two subunits of the actin related protein $2 / 3$ complex (ARP2/3) and the GTP binding proteins Dnm2 and Rac1. Along with Rac1, the Rac1-activated kinase Pak1 was also highlighted as a potential regulator of the internalisation of Tie2.

It was especially relevant to find Rac1 as a robust potential modulator of the internalisation of Tie2, as Rac1 is activated by the signalling cascade triggered by Tie 2 phosphorylation and it is implicated in physiological and cancer angiogenesis. This result highlights a potential cross-talk between the endocytosis and signalling of Tie2 that we believe needs to be further investigated. 